\title{
CT angiography of renal arteries: anatomy, variants and its importance in the living donor.
}

\author{
Kolla Praveen ${ }^{1}$, Pentyala Suneetha ${ }^{2}$, Lattupalli $\mathrm{Hema}^{3}$, \\ ${ }^{I}$ Professor of Nephrology, Narayana Medical College, Nellore. \\ ${ }^{2}$ Professor of Radiology, Narayana Medical College, Nellore. \\ ${ }^{3}$ Professor and HOD, Department of Anatomy, Narayana Medical College, Nellore.
}

\begin{abstract}
:
Background: Kidneys are retroperitoneal organs normally supplied by the paired renal arteries. Living donor renal transplantation, as opposed to cadaveric donation, is the best opinion for the recipient and graft survival. The need for kidney transplantation has undergone exponential growth over the past 40 years, and cadaveric donations are inadequate to meet this ever-increasing demand. These factors have led to a continued increase in the organ donation from living related donors.
\end{abstract}

Aim of the study: The purpose of this present study is to illustrate the MDCT angiographic appearance of normal anatomy and common variants of renal vasculature in Indian population and also to provide their embryological and clinical correlation.

Material and methods: 100 living donor cases were taken for the study. The normal and variant renal vasculature was studied by using multidetector computed tomography angiography 128 slice (MDCTA).

Observations: In 100 (100\%) cases that were taken for the study 22 cases (22\%) showed variations in the bifurcation and branching of the renal arteries was observed. There was an early bifurcation of the renal artery in 10 cases $(45.45 \%)$. There was an accessory renal artery bilateral in 12 cases $(54.54 \%)$.

Conclusion: Identification of renal vascular variants is important, especially before laparoscopic donor or partial nephrectomy and vascular reconstruction for renal artery stenosis or abdominal aortic aneurysm. MDCT angiography is an excellent imaging investigation because it is a fast and non-invasive tool that provides highly accurate and detailed evaluation of normal renal vascular anatomy and variants. The number, size and course of the renal arteries and veins are easily identified by MDCT angiography.

Keywords: Living donor, Multidetector computed tomography, Renal artery, Variations, Embryology, Anatomy.

\section{Introduction}

Kidneys are a pair of retroperitoneal organs supplied by a single renal artery, which arises as a lateral branch of abdominal aorta, between the levels of L1 and L2 (BEREGI, MAUROY, WILLOTEAUX et al., 1999; OZKAN, OGUZKURT, TERCAN et al., 2006).However the classical description of the renal vasculature, formed only by one artery and one vein, occurs in less than 25\% of cases (AWOJOBI, OGUNBIYI and NKPOSONG, 1983; CIÇEKCIBASI, ZIYLAN, SALBACAK et al., 2005).Most often encountered morphological variations of renal artery are its variable number and unusual branches originating from it (DHAR and LAL, 2005; RUSU, 2006; SHOJA, TUBBS, SHAKERI et al., 2008a). Most of these variations remained undiscovered until being noticed during any surgical procedure or found by forensic pathologist during autopsy (KRISHNASAMY, RAO, SOMAYAJI et al., 2010). Variations in renal arteries have been called aberrant, supernumerary (Merklin classification), supplementary, accessory, among other terms. It is therefore necessary that the morphology and the nomenclature of these vessels are standardized. According to Sampaio and Passos (1992) these arteries should be called multiple, since they are segmental vessels for the kidneys, without anastamosis between themselves and they should be named according to the territory supplied by them as- hilar, superior polar and inferior polar.

The study of the renal pedicle and its peculiarities has been of interest to anatomists since the end of the nineteenth century. The distribution and morphology of these anomalies can be explained by considering the embryological development of the renal vasculature, but it was after the outburst of imaging techniques that radiologists started recognizing and describing unconventional renal vascular patterns, which led to a systematic clinical approach to this topic. Knowledge of these patterns has gained significance with the advent of the era of transplantation. Given this growing demand, kidneys from living donors have been considered as an alternative to cadaveric donation to expand the pool of organs. Living kidney donation demonstrates many advantages and stands out as the best alternative for organ procurement. There has been a significant reduction of waiting time for transplantation since it can be done on a scheduled 
basis, even before entry into dialysis programmes [1]. When performed in this manner, an optimization of conditions of donor and recipient, such as minimum ischemia time, can be achieved. There is a lower incidence of delayed graft function, which is a negative prognostic factor for graft survival [1].

This fact allows not only for better graft endurance but also superior recipient survival compared to transplantation with a cadaveric donor. There is a reduced incidence of rejection and therefore less need for immunosuppressive drugs. As a consequence, not only more organs are available, but also there is less need for retransplantation. Furthermore, live donor transplantation is more cost effective than cadaveric transplantation [1].Multidetector computed tomography angiography (MDCTA) plays an increasingly important role in the evaluation of the renal vasculature (1). Although conventional angiography is still regarded as the gold standard in renal vascular imaging, MDCTA is increasingly used as it is less invasive, easily applicable and available. MDCTA enables precise visualization of the normal and variant anatomy of several regions including the renal vasculature; however, the main drawbacks of MDCTA are the exposure to ionizing radiation and the use of potentially nephrotoxic iodinated contrast material. As such, its use is limited in children and pregnant women and in patients with impaired renal function.

Main clinical indications for renal MDCTA include the imaging workup for ruling out renovascular hypertension, renal transplant recipient

and donor evaluation, acute onset flank pain in patients with coagulative disorders, direct renal trauma, arteriovenous communications, renal artery aneurysm, renal parenchymal or vascular calcifications, renal manifestations of a systemic disease (e.g., vasculitis, thromboembolic disease).

\section{Materials and Methods}

100 living donor cases were taken for the study. The normal and variant renal vasculature was studied by using multidetector computed tomography angiography 128 slice (MDCTA).

MDCT angiography was performed by using a 128 slice MDCT system (GE Medical Systems, Milwaukee, Wis., USA) with the protocol acquiring CT data in the arterial and nephrographic phases. To minimize the dose of ionizing radiation, we do not usually acquire unenhanced or venous phase images. Firstly, an initial scout image was obtained. Subsequently, $100-140 \mathrm{ml}$ of nonionic iodinated contrast agent (Iodixanol, Visipaque 320 mg I/ml, GE Healthcare, Milwaukee, Wis.) was injected through an 18-gauge cannula positioned in an antecubital vein at a flow rate of $4 \mathrm{ml} / \mathrm{s}$ by using a power injector. The estimated dose was determined on the basis of patient weight as follows: weight of less than $45 \mathrm{~kg}, 100 \mathrm{ml} ; 45-90 \mathrm{~kg}, 120 \mathrm{ml}$; and greater than $90 \mathrm{~kg}$, $150 \mathrm{ml}$.The start time of the arterial phase acquisition was determined using automatic bolus tracking (Smart Prep,GE Healthcare) $5 \mathrm{~s}$ after a threshold of $125 \mathrm{HU}$ was reached in a region of interest within the abdominal aorta just cranial to the kidneys. This automatic bolus tracking meant that the arterial phase acquisition began 18-27 s after the start of the injection. The main acquisition parameters forthe arterial phase were: the detector collimation of $16 \times 0.625 \mathrm{~mm}$, tube voltage of $120 \mathrm{kv}$, tube current of $200-240 \mathrm{mAs}$, gantry speed of 0.5 s/rotation. Nephrographic phase images were then acquired $85 \mathrm{~s}$ after the start of the injection of contrast medium with 2.5 -mm collimation. The area scanned extended from diaphragm to midsacrum. All images were reconstructed with a standart soft tissue algorithm and transferred to a separate workstation for post-processing. For three-dimensional image reconstruction, the volumetric MDCT data sets were processed on a separate workstation (Advanced Workstation 4.2, GEHealthcare, Milwaukee, Wis.) with multiplanar reformatting, curved planar reformatting, maximum intensity projection and volume rendering. For three-dimensional MDCT angiography, volume rendering techniques were usually used, but multiplanar and maximum intensity projection images were also used, especially for evaluation of the venous system or small arteries. Renal arterial and venous anatomy was assessed primarily on arterial phase images, but if the renal veins, especially accessory draining renal veins including lumbar and gonadal veins, were not enhanced on the arterial phase images, nephrographic phase images were used.

Observations: In $100(100 \%)$ cases that were taken for the study 22 cases $(22 \%)$ showed variations in the bifurcation and branching of the renal arteries was observed. There was an early bifurcation of the renal artery in 10 cases $(45.45 \%)$. There was an accessory renal artery bilateral in 12 cases $(54.54 \%)$.

\section{Normal Renal Arterial Anatomy And Variants}

Renal arteries typically arise from the aorta at the level of the superior margin of the second lumbar vertebral body, slightly inferior the origin of the superior mesenteric artery. The right renal artery orifice is usually more superior and anterolateral than the left. Typically, the right renal artery has a long downward course to the relatively inferior right kidney, whereas the left renal artery has a more horizontal course to the superiorly located left kidney. The main renal arteries divide into anterior and posterior divisions that lie anterior and posterior to the renal pelvis. The anterior division branches into four segmental arteries including apical, upper, middle, and lower anterior. The apical and lower anterior segmental arteries supply the anterior and posterior surfaces of the upper and lower renal poles, and the upper and middle segmental arteries supply the 
remainder of the anterior surface. The posterior division supplies a large portion of the blood flow to the posterior portion of the kidney. The segmental arteries course through the renal sinus and further subdivide into interlobar arteries. At the level of the renal pyramids the interlobar arteries divide into arcuate arteries, which parallel the renal contour along the corticomedullary junction. The arcuate arteries give rise to multiple interlobular arteries. Also, the renal arteries give off inferior adrenal branches, capsular branches, and branches into renal pelvis and proximal ureter.

About $70 \%$ of the population may be expected to have a single renal artery that originates from the abdominal aorta on each side.

\section{Discussion}

Accessory renal arteries are the most common renal vascular variant and are seen in about one-third of the population. In a study of 400 cadaver renal donors with 800 kidneys, Pollak et al. detected that $23 \%$ had double renal arteries $4 \%$ triple renal arteries and $1 \%$ quadruple renal arteries. Bilateral multiple renal arteries occur in $10-15 \%$ of the population. Accessory renal arteries are considered to be persistent embryonic lateral splanchnic arteries. The origins of the accessory renal arteries may be a high or low position of the abdominal aorta when it originates from a low position, its origin may be near the aortic bifurcation or from the iliac arteries. Most commonly, the accessory arteries originate from the abdominal aorta and supply the inferior pole of the kidney. Rarely, they can arise from the coeliac, mesenteric, lumbar, middle colic or middle sacral artery. Accessory renal arteries are categorized according to their course as either polar (piercing the upper or lower pole of the kidney directly) or hilar (entering the kidney at the hilum). The polar accessory renal arteries are usually smaller but hilar accessory renal arteries are not always smaller than the principal renal arteries. In the present study in 100 cases that were taken for the study 22 cases (22\%) showed variations in the bifurcation and branching of the renal arteries was observed. There was an early bifurcation of the renal artery in 10 cases $(45.45 \%)$. There was an accessory renal artery bilateral in 12 cases $(54.54 \%)$

Prehilar (early) branching of the renal artery is a normal variant in which any branch diverge within $1.5-2.0 \mathrm{~cm}$ from the lateral wall the aorta in the left kidney or in retrocaval segment in the right kidney. This variant is important in renal transplantation, because most surgeons usually require at least a $1.5-$ to $2.0-\mathrm{cm}$ length of renal artery before first branching for successful anastamosis.

Embryological explanation of these variations has been presented and discussed by Felix [18]. In an 18 $\mathrm{mm}$ fetus, the developing mesonephric, metanephros, suprarenal glands, and gonads are supplied by nine pairs of lateral mesonephric arteries arising from the dorsal aorta. Felix divided these arteries into three groups as follows: the $1^{\text {st }}$ and $2^{\text {nd }}$ arteries as the cranial, the $3^{\text {rd }}$ to $5^{\text {th }}$ arteries as the middle, and the $6^{\text {th }}$ to 9 th arteries as the caudal group. The middle group gives rise to the renal arteries. Persistence of more than one artery of the middle group results in multiple renal arteries [18]. Thus, the multiple renal arteries in our study are a result of persisting lateral mesonephric arteries from the middle group.

Clinically, the supernumerary renal arteries are very important. Upper polar and lower polar supernumerary renal arteries originating from renal arteries, directed towards superior or inferior pole, have vertical trajectory in comparison to supernumerary renal arteries taking origin from aorta. Vertical trajectory of these arteries can lead to polar infarction [19] and they can also be injured during mobilization and other surgical procedures [22]. Lower polar supernumerary renal arteries of aortic or renal origin can be a cause of uretero pelvic junction obstruction [20].

The anatomical knowledge of supernumerary renal arteries is essential before performing any transplantation surgeries where micro vascular techniques are employed to reconstruct the renal arteries [21]. One has to keep in mind that transplanting a kidney with accessory renal arteries has several theoretical disadvantages: acute tubular necrosis and rejection episodes and decreased graft function [22].

We believe that awareness of variations is necessary for surgical management during renal transplantation, repair of abdominal aorta aneurysm, and urological procedures and for angiographic interventions.

References

[1]. Khamanarong K, Prachaney P, Utraravichien A, Tong-un T, Sripaoraya K (2004) Anatomy of renal arterial supply. Clin Anat $17: 334-336$.

[2]. Hänninen EL, Denecke T, Stelter L,Pech M, Podrabsky P, Pratschke J et al (2005) Preoperative evaluation of living kidney donors using multirow detector computed tomography: comprasion with digital substraction angiography and intraoperative findings. Transpl Int 18:1134-1141

[3]. Rastogi N, Sahani DV, Blake MA, KoDC, Mueller PR (2006) Evaluation of living renal donors: accuracy of three-dimensional 16section CT. Radiology 240:137-144

[4]. Raman SS, Pojchamarnwiputh S,Muangsomboon K, Schulam PG,Gritsch HA, Lu DSK (2007) Surgically relevant normal and variant renal parenchymal and vascular anatomy in preoperative 16-MDCT evaluation of potential laparoscopic renal donors.AJR Am J Roentgenol 188:105-114

[5]. Rydberg J, Liang Y, Teague SD (2003) Fundamentals of multichannel CT. Radiol Clin North Am 41:465-474 
[6]. Monroy-Cuadros M, McLaughlin K,Salazar A, Yilmaz S (2008) Assessment of live kidney donors by magnetic resonance angiography: reliability and impact on outcomes. Clin Transplant22:29 - 31

[7]. Beregi JP, Mauroy B, Willoteaux S,Mounier-Vehier C, Remy-Jardin M, Francke J (1999) Anatomic variation in the origin of the main renal arteries: spiral CTA evaluation. Eur Radiol 9:1330 - 1334

[8]. Kadir S (1986) Angiography of the kidneys. In: Kadir S (ed) Diagnostic angiography. Saunders, Philadelphia, pp 445-495 9. Pollak R, Prusak BF, Mozes MF (1986) Anatomic abnormalities of cadaver kidneys produced for purposes of transplantation. Am Surg 52:233-235

[9]. Satyapal KS, Haffejee AA, Singh B,Ramsaroop L, Robbs JV, Kalideen JM (2001) Additional renal arteries: incidence and morphometry. Surg Radiol Anat 23:33-38

[10]. Williams PL, Warwick R, Dyson M,Bannister LH (1989) The urinary organs. In: Williams PL, Warwick R,Dyson M, Bannister LH (eds) Gray'sanatomy, 37th edn. Churchill Living-stone, New York, pp 1397-1416

[11]. Urban BA, Ratner LE, Fishman EK(2001) Three-dimensional volume-rendered CT angiography of the renal arteries and veins: normal anatomy, variants, and clinical applications. Radiographics 21:373-386

[12]. Abrams HL (1983) Renal venography.In: Abrams HL (ed) Abrams angiogra- phy, 2nd edn. Little Brown, Boston, pp1327-1364

[13]. 14. Trigaux JP, Vandroogenbroek S, DeWispelaere JF, Lacrosse M, Jamart J (1998) Congenital anomalies of the inferior vena cava and left renal vein: evaluation with spiral CT. J Vasc Intervent Radiol 9:339-345

[14]. Kawamoto S, Lawler LP, Fishman EK (2005) Evaluation of the renal venous system on late arterial and venous system on late arterial and venous phase images with MDCT angiography in potential living laparoscopic renal donors. AJR Am J Roentgenol $184: 539-.545$

[15]. KRISHNASAMY, N., RAO, M., SOMAYAJI SN., KOSHY, S. and RODRIGUES, V. An unusual case of unilateral additional right renal artery and vein. International Journal of Anatomical Variations, 2010, vol. 3, p. 9-11

[16]. AWOJOBI, OA., OGUNBIYI, OA. and NKPOSONG, EO. Unusual relationship of multiple renal arteries. Urology, 1983, vol. 21, n. 2, p. 205-206. 18.W. Felix, "Mesonephric arteries (aa. mesonephrica)," in Manual of Human Embryology, F.KeibelandF.P.Mall,Eds.,vol.22,pp.820-825, Lippincott, Philadelphia, Pa, USA, 1912.

[17]. R. W. Beyer and P. O. Daily, "Renal Artery Dissection Associated with Gz Acceleration, "Aviation Space and Environmental Medicine, vol.75,no.3,pp.284-287,2004.

[18]. M. M. Shoja, R. S. Tubbs, A. Shakeri, M. R. Ardalan, B. Rahimi-Ardabili, and K. Ghabili, "Asymptomatic bilateral uretero pelvic junction obstruction due to supernumerary renal arteries, "Saudi Journal of Kidney Disease and Transplantation ,vol.19,no.5,pp.806-808,2008.

[19]. G. E. Brannen, W. H. Bush, and R. J. Correa, "Micro vascular management of multiple renal arteries in transplantation, "Journal of Urology ,vol.128,no.1,pp.112-115,1982.

[20]. F.J.B.Sampaio and M.A.R.F.Passos, "Renal arteries: anatomic study for surgical and radiological practice, ”Surgical and Radiologic Anatomy, vol.14,no.2, pp.113-117,1992

[21]. CIÇEKCIBASI, AE., ZIYLAN, T., SALBACAK, A., SEKER, M., BUYUKMUMCU, M. and TUNCER, I. An investigation of the origin, location and variations of the renal arteries in human fetuses and their clinical relevance. Annals of anatomy, vol. 187, 2005, n. 4, p. 421-427.

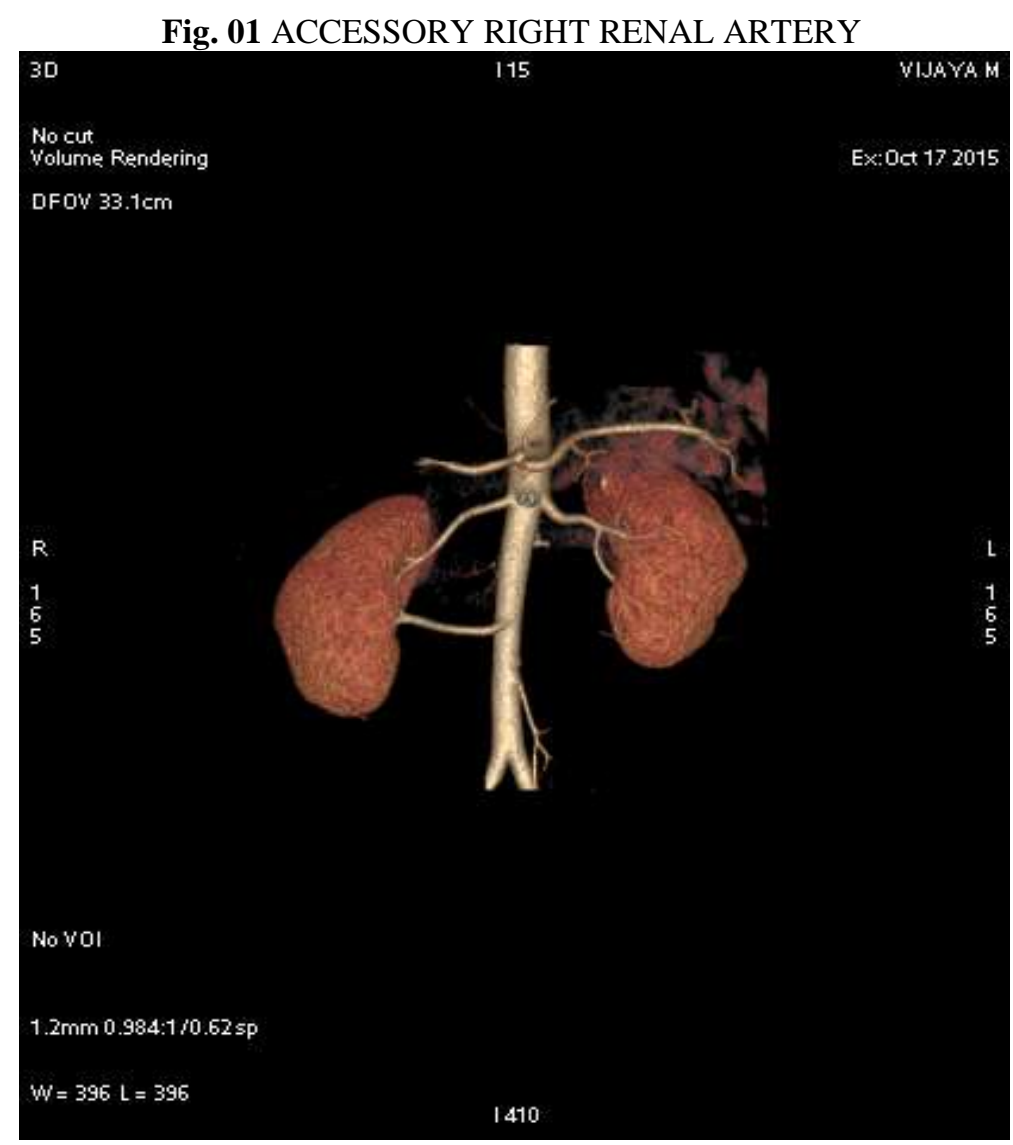




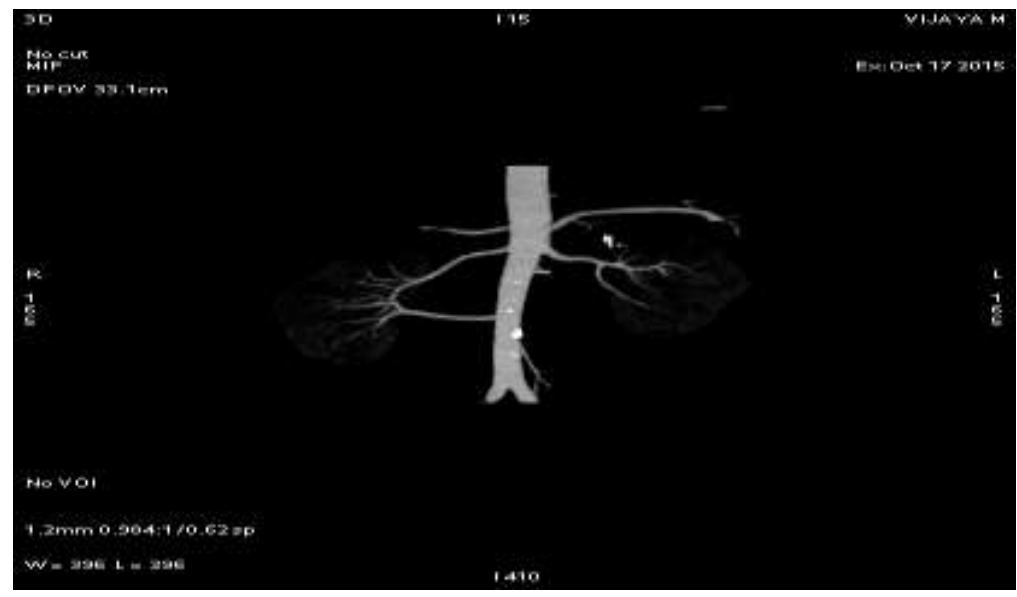

Fig. 2 Early Bifurcation Of Right Renal Artery.
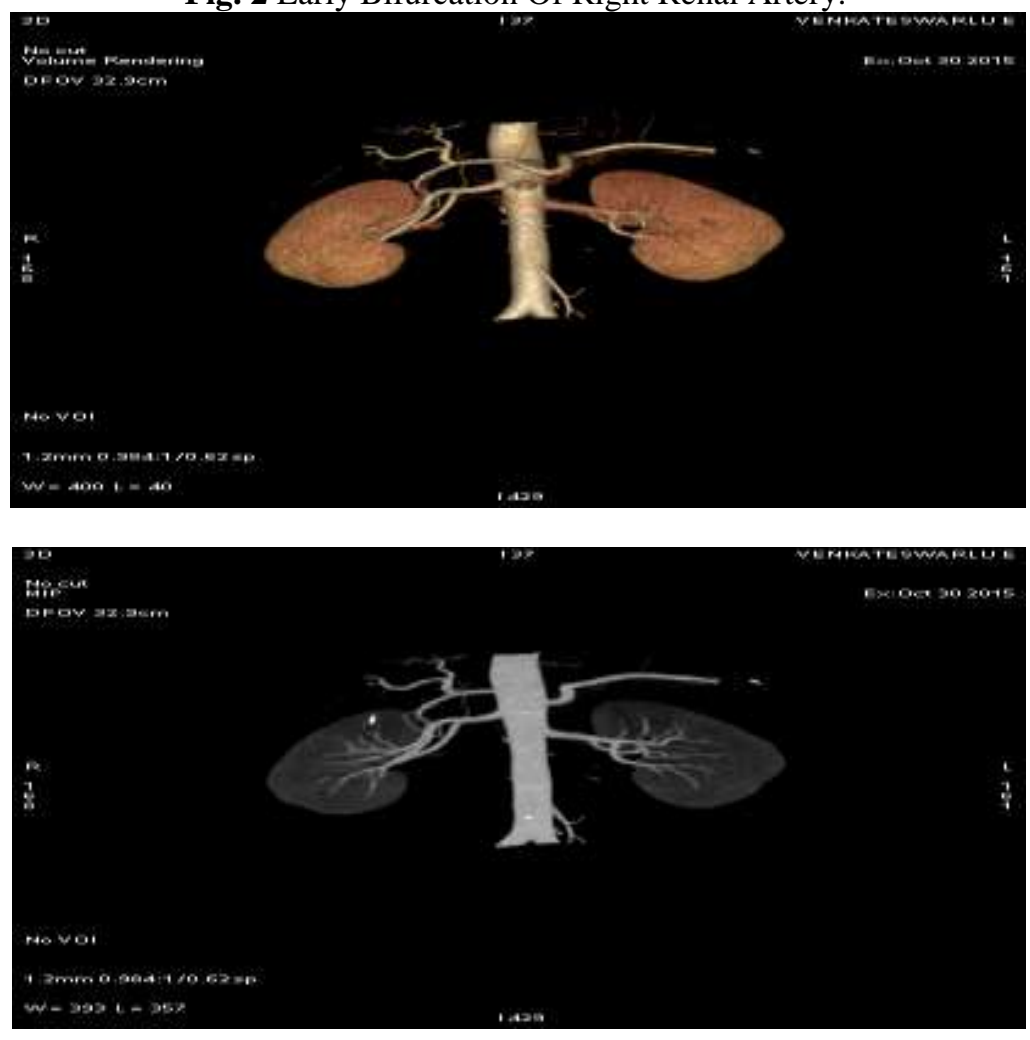

Fig. 03 Accessory Renal Artery In Left Kidney. Accessory Renal Vein In Left Kidney.

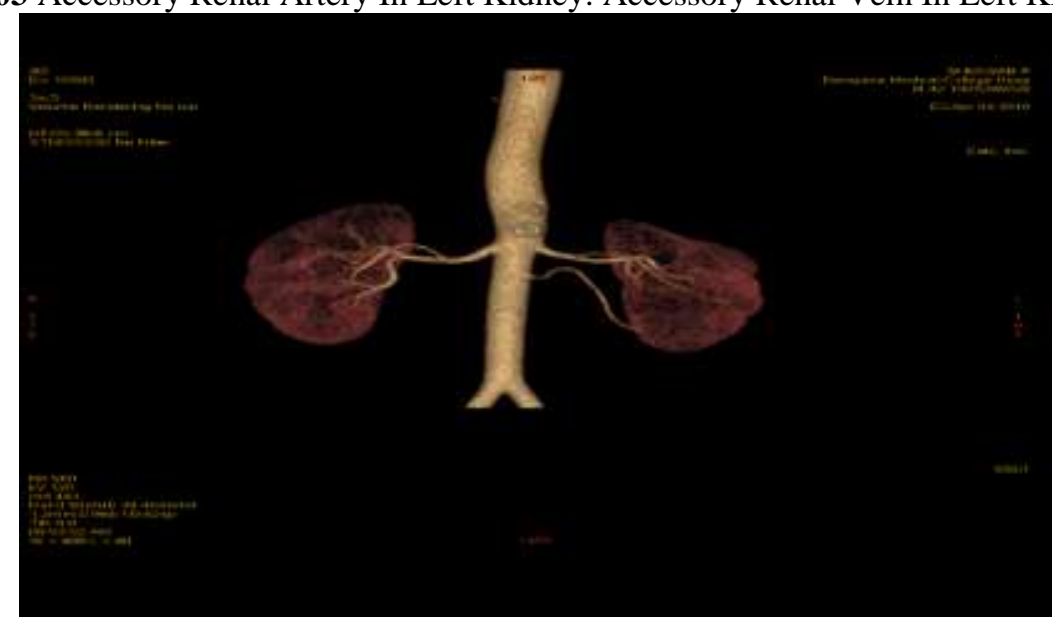


CT Angiography of Renal Arteries: Anatomy, Variants And Its Importance In The...
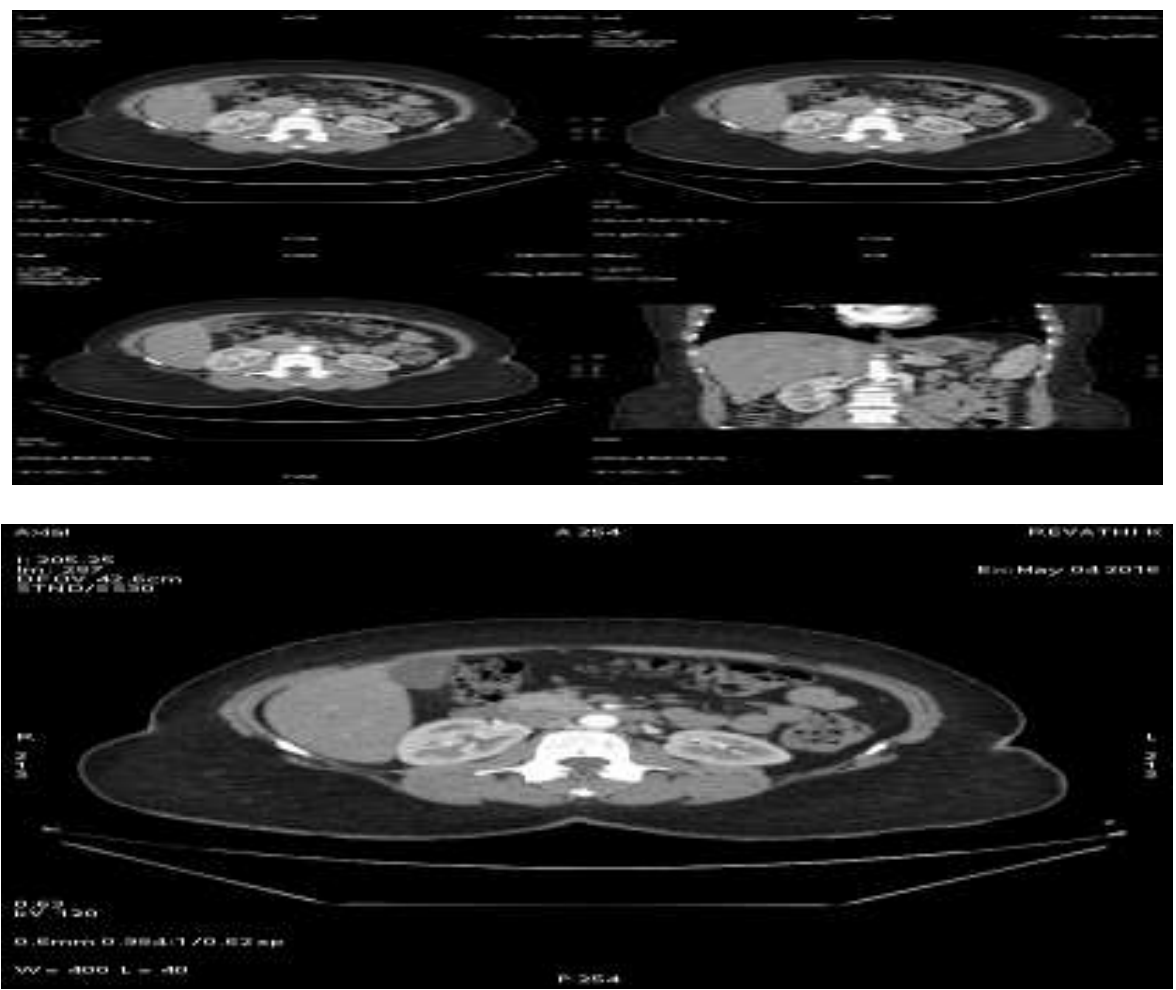

Retroaortic Renal Vein

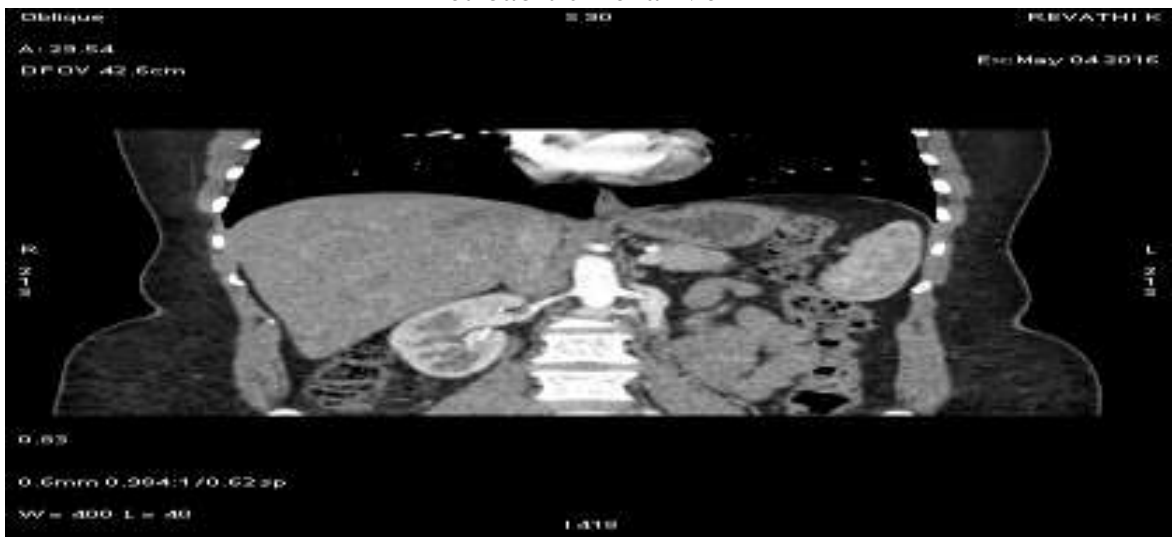

